\title{
COMMENTARIES
}

\section{UNDERSTANDING THE VARIABILITY IN ULTRAFILTRATION OBTAINED WITH ICODEXTRIN}

$\mathrm{I}_{\mathrm{a}, \mathrm{s}}^{\mathrm{cos}}$ codextrin (ICO) has been a major addition to the management of ultrafiltration (UF) in peritoneal dialysis (PD), allowing sustained UF during long dwells and maintenance of UF in the face of increased membrane solute transport, while minimizing the deleterious effects of local and systemic glucose exposure. Initial studies indicated ICO typically achieved UF over $500 \mathrm{~mL}$ (1-4), but these studies were mostly using the overnight dwell of continuous ambulatory PD and may not always have taken overfill into account. An increasing number of studies are reporting greater variation in achieved UF, particularly in automated PD patients that use a longer dwell (3-5). This runs counter to the UF with ICO previously predicted by computer-generated three-pore modeling, which suggested UF should increase linearly up to at least 17 hours. Given the importance of UF and the widespread use of ICO, this is an area requiring further clarification.

\section{WHAT IS THE EVIDENCE FOR VARIABILITY OF UF WITH ICO?}

This variability was first noted by us in 2004 when analyzing 9-hour dwells: a median volume of $370 \mathrm{~mL}$ UF was observed but with significant variation between individuals, with volumes ranging from $>500 \mathrm{~mL}$ of net fluid reabsorption to $>1000 \mathrm{~mL}$ UF. Some variation within individuals was also noted but to a lesser degree than the between-patient variation.

The factors underlying this variation remain unclear and the study by Jeloka et al. (6), on which Venturoli et al.'s work published in this issue of Peritoneal Dialysis International (7) was based, attempted to investigate some of these factors. Canadian and Turkish PD patients $(n=36)$ were given ICO in gradually increasing dwell lengths (from 10 to 14 hours). Mean achieved UF ranged from $352 \mathrm{~mL}$ at 10 hours to $390 \mathrm{~mL}$ at 13 hours but the standard deviation was consistently around $250 \mathrm{~mL}$, with the minima being $>300 \mathrm{~mL}$ reabsorption and the maxima $1012-1240 \mathrm{~mL}$. There was no correlation with dwell length and the only significant finding was an association between increased mean UF and either male gender or high transport characteristics. Median within-patient standard deviations were $104 \mathrm{~mL}$ whereas the betweenpatient standard deviations were $267 \mathrm{~mL}$, confirming the earlier observation that there is a high degree of between-patient variability in UF achieved with ICO.

\section{WHAT FACTORS COULD BE AFFECTING UF WITH ICO?}

The three-pore model is currently the only mathematical description of membrane function that can provide both an accurate explanation for and a prediction of the UF achieved with either glucose or ICO dialysis fluids. Furthermore, the model is also able to predict UF profiles when ICO is combined with glucose. For example, computer-based modeling with the three-pore model was used to suggest different concentrations of the combination that would result in improved UF, and was subsequently shown experimentally to be correct $(8,9)$.

According to the three-pore model, ICO will, through its colloid osmotic pressure (COP), act across small pores to achieve UF with negligible effect across the ultrasmall pores (aquaporins). Factors that could potentially affect the degree of UF will therefore include the number of small pores (broadly equivalent to membrane transport status), the UF coefficient of the membrane (LpS), and factors affecting the colloidal concentrations on either side of the membrane, that is, plasma COP and the metabolism or clearance of ICO itself. Additional factors that will also affect the net achieved volume of UF will include the intraperitoneal hydrostatic pressure opposing the COP gradient, the "lymphatic" reabsorption rate, and, importantly, the presence of any leaks from the abdominal cavity. 


\section{WHAT EFFECT COULD MEMBRANE FACTORS HAVE?}

According to the three-pore model, "high transport status" corresponding to a high permeability-surface area product (PS) - also known as mass transfer area coefficient (MTAC) - represents a greater capillary surface area and therefore a larger small pore area. As ICO's main effect on UF is across the small pores, this means that, not only should patients with a high transport status achieve a better UF with ICO versus glucose, but that they should achieve a greater UF with ICO than would patients with low transport status with ICO. The former point is well established but there is also evidence for the latter, with both our observations reported here and Jeloka's work demonstrating a greater UF with high transport status. However, only a small amount of UF variation could be attributed to this in both cases, indicating that transport status has only a minor role in determining the variability of UF with ICO.

Hydraulic permeability (Lp) is a measure of the membrane's permeability to water per unit area and, when multiplied by surface area, this gives LpS (also called LpA), commonly called the UF coefficient. There is increasing evidence that the more severe type $2 \mathrm{UF}$ failure seen after prolonged PD, associated not just with high solute transport but also with reduction in the osmotic conductance of the membrane (10) and thought to be associated with the development of fibrosis (11), could be due to reduced LpS. This could be the explanation of our own observation that the net achieved UF with ICO is less in long-term PD patients (see Figure 1). However, no studies to date have correlated LpS with UF from ICO, but this has clear potential to be one of the sources of variation and warrants further investigation.

\section{WHAT EFFECT COULD DIFFERENT HYDROSTATIC AND OSMOTIC PRESSURES HAVE?}

While the intrinsic characteristics of the membrane will affect how efficiently water flows through it, the balance of the hydrostatic, crystalloid, and colloid pressure gradients determine the quantity and direction of flow. The formulation of icodextrin PD solution is isoosmotic with plasma so, unless there is significant intraperitoneal metabolism (see below), this should have little effect. An elevated intraperitoneal hydrostatic pressure will favor fluid reabsorption by whatever route (transcapillary or lymphatic), potentially increasing as the volume of fluid within the peritoneum increases. Several studies have shown hydrostatic pressure to have a significant effect on net UF with glucose exchanges $(12,13)$. One study in children comparing the effect of

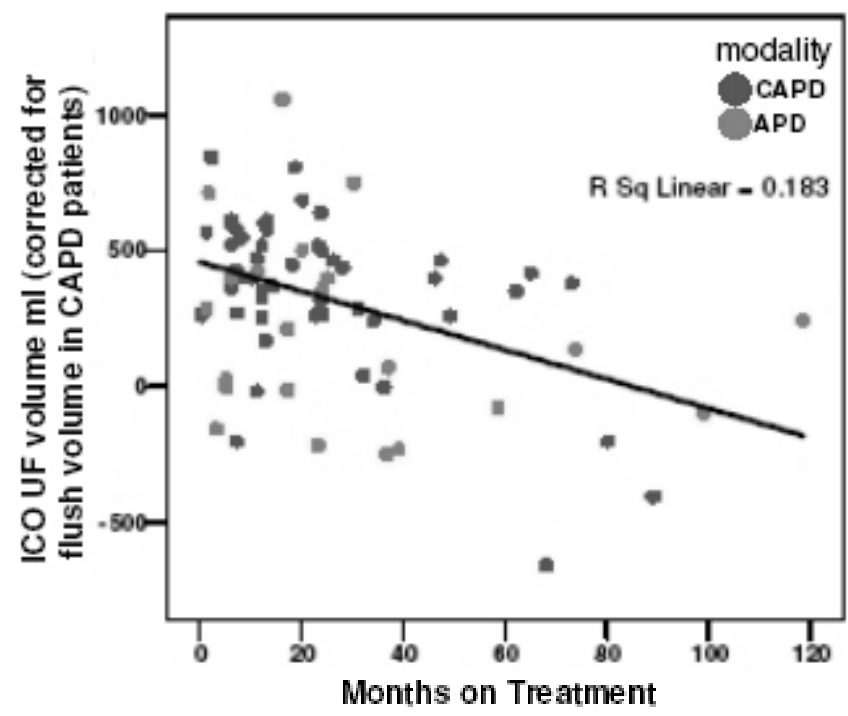

Figure 1 - Net ultrafiltration achieved over 9 hours in CAPD (corrected for overfill) and APD patients according to length of time on treatment. Multivariate analysis indicated that length of time on treatment was associated with less $(p<$ 0.001 ), whereas high solute transport was associated with more ultrafiltration $(p=0.031)$. ICO = icodextrin; UF = ultrafiltration; $C A P D=$ continuous ambulatory peritoneal dialysis; APD = automated PD. (Stompor and Davies; previously published in abstract form only).

intraperitoneal pressure on net UF achieved with glucose or ICO confirmed the negative association with the former but found no relationship with the latter (14). However, these results should be interpreted with caution, especially when extrapolating to adults, as observations were limited to 4-hour dwells and it is known that children achieve less UF with ICO than seen in the adult population (15).

As ICO exerts its main effects by creating a COP gradient across the peritoneum, it follows that this will, in turn, be affected by the intracapillary COP exerted by plasma proteins. The relationship between protein concentration and COP is not straightforward - albumin possesses approximately twice the COP per gram compared with globulins - and several equations have been described to predict COP from either total protein or albumin. One equation has been derived for nephrotic patients using both albumin and fibrinogen (16) but there have been no studies examining the true plasma COP in PD patients, a group known to have abnormal protein kinetics with dialytic protein loss.

Plasma COP has been noted to vary diurnally, with hemolysis, with recumbence, and with fasting $(17,18)$. Changes in intravascular volume, which may reflect extracellular volume, will also affect plasma COP as with 
more volume there is more water to dilute the proteins and decrease the plasma COP. Theoretically, patients with fluid overload could achieve better UF with ICO. This is supported by the randomized trials investigating the effect of ICO on fluid status where the greatest improvement was seen in the most overhydrated patients at the start of the studies $(19,20)$. Conversely, with a greater degree of UF, extracellular volume will contract, potentially inhibiting further UF by producing a higher plasma COP, although plasma albumin concentration did not change in these intervention studies. However, the effect of plasma COP on UF has not been examined and this could possibly explain some of both the intra- and the interindividual variation.

\section{WHAT EFFECTS COULD THE METABOLISM AND CLEARANCE OF ICO HAVE?}

Rates of metabolism and clearance of ICO could affect UF in two ways. First, metabolites of ICO are found in the plasma, thereby potentially increasing the crystalloid osmotic pressure of plasma and opposing UF out of the capillaries. Second, clearance of ICO will decrease the intraperitoneal COP by which ICO exerts its effect on UF.

Icodextrin is a mix of different length polymers of glucose whose primary route of degradation is thought to be by plasma amylase producing shorter polymers. These shorter polymers are then metabolized by tissue maltases, which break these short polymers down to glucose. Amylase levels are high in predialysis, hemodialysis, and PD patients on glucose-only dialysis but are strikingly reduced in patients on ICO. This discrepancy was previously thought to reflect interference with the assay by ICO metabolites present in the plasma but more recent evidence suggests this is not necessarily the case (21) and the reason remains unclear. The overall impact of plasma amylase and low molecular weight ICO metabolites in plasma was investigated in a secondary analysis of a randomized controlled trial that demonstrated no correlation between either of these and changes in fluid status (20).

Clearance of ICO from the peritoneal cavity follows zero-order kinetics, with the amount absorbed being directly proportional to the dwell length, which is consistent with convective flow. At 8 hours, approximately $20 \%$ of ICO has been cleared, and at 16 hours, $40 \%$. This does not specify the route of clearance, which will be partially through lymphatics but could also include penetration into the interstitium, where it may be metabolized, and leaks by any anatomic route. The true proportion of clearance via these different pathways is unclear as their direct measurement is technically difficult.

The frequency of leaks causing UF problems with ICO is unclear but a recent presentation at an ISPD conference reported an investigation into causes of acute UF failure where all patients with this problem had computerized tomographic or magnetic resonance imaging of their abdomen: $64 \%$ of these patients had evidence of a retroperitoneal leak and this leak was associated with a high odds ratio, compared with other PD patients, of a hernia or pleuroperitoneal leak (22). While this study requires confirmation in other populations, it emphasizes an important clinical issue where UF failure should prompt a high index of suspicion for a leak.

\section{WHAT DOES THE ANALYSIS BY VENTUROLI ADD TO OUR} UNDERSTANDING OF THIS COMPLEX PROBLEM?

Venturoli et al. investigated some of the possible reasons for variation in UF with ICO by further analyzing the work by Jeloka et al. Initially, using linear and quadratic regression, they identified individual patterns in UF over 10- to 14-hour dwells. Four profiles were described: steadily increasing, steadily decreasing, concave, and convex. They then sought to fit these patterns to the three-pore model by adjusting different factors, including UF coefficient (LPS), clearance of large molecular weight fractions of ICO from the peritoneal cavity $\left(\mathrm{Cl}_{\mathrm{LF}}\right)$, diffusivity of the membrane (PS or MTAC), and transcapillary COP gradient and, using the three-pore model, predicted UF curves. The steadily increasing and concave curves were readily predicted with variations in UF coefficient and diffusivity. The best fit for the steadily decreasing curve came from variations in the transcapillary COP gradient, whereas the convex curve was not predicted by any of the changes. In particular, this process highlighted the large variation in UF predicted from relatively minor adjustments in the transcapillary COP gradient. The authors did not investigate the effect of varying true lymphatic fluid reabsorption, although this could potentially lead to variability in net UF.

The Venturoli study certainly sheds light on the way in which we need to consider the issue of UF with ICO. Whereas UF was previously considered to follow a simple linear increase with dwell length, with any variation being due simply to differences in the gradient, this is clearly not the case, as the four different patterns between 10 and 14 hours demonstrated. The three-pore model has again illustrated its utility by describing three of these patterns, so suggesting possible mechanisms. The wide variation in UF shown in previous studies could 
therefore be partly explained by differences in plasma COP, macromolecular clearance, and LpS, although there is still some unexplained variation. Figure 1 in Venturoli's paper demonstrates well the intraindividual variation that this study has not explained, although it has highlighted the potential importance of plasma COP, a factor known to vary within individuals.

\section{HOW DOES THIS AFFECT OUR CLINICAL PRACTICE?}

As most of the Venturoli paper is based on theoretical modeling, empirical validation remains crucial. For the time being, however, clinicians can no longer assume that UF will simply increase with time and consideration must now be given to the optimal length of dwell that each patient uses for ICO. Any patient exhibiting net fluid reabsorption with ICO should be closely investigated for a peritoneal leak. Until we have a predictive model based on sound clinical validation from which an "ICO peritoneal equilibration test" can be developed, establishing the optimal dwell length will involve nothing more sophisticated than trial and error.

\section{DISCLOSURE}

Simon Davies receives research funding from and is an Advisory Board member for Baxter Healthcare.

Mark Lambie ${ }^{1,2}$
Tomasz Stompor
Simon Davies
1,2*

University Hospital of North Staffordshire ${ }^{1}$ Institute for Science and Technology in Medicine ${ }^{2}$ Keele University, Stoke-on-Trent, UK Department of Nephrology ${ }^{3}$ Jagiellonian University, Kraków, Poland

*e-mail: SimonDavies1@compuserve.com

\section{REFERENCES}

1. Mistry $C D$, Gokal R, Peers E. A randomized multicenter clinical trial comparing isosmolar icodextrin with hyperosmolar glucose solutions in CAPD. MIDAS Study Group. Multicenter Investigation of Icodextrin in Ambulatory Peritoneal Dialysis. Kidney Int 1994; 46:496-503.

2. Johnson DW, Arndt M, O'Shea A, Watt R, Hamilton J, Vincent K. Icodextrin as salvage therapy in peritoneal dialysis patients with refractory fluid overload. BMC Nephrol 2001; 2(1):2.

3. Wolfson M, Piraino B, Hamburger RJ, Morton AR. A randomized controlled trial to evaluate the efficacy and safety of icodextrin in peritoneal dialysis. Am J Kidney Dis 2002; 40:1055-65.

4. Finkelstein F, Healy H, Abu-Alfa A, Ahmad S, Brown F, Gehr $\mathrm{T}$, et al. Superiority of icodextrin compared with $4.25 \%$ dextrose for peritoneal ultrafiltration. J Am Soc Nephrol 2005; 16:546-54.

5. Woodrow G, Stables G, Oldroyd B, Gibson J, Turney JH, Brownjohn AM. Comparison of icodextrin and glucose solutions for the daytime dwell in automated peritoneal dialysis. Nephrol Dial Transplant 1999; 14:1530-5.

6. Jeloka TK, Ersoy FF, Yavuz M, Sahu KM, Camsari T, Utas C, et al. What is the optimal dwell time for maximizing ultrafiltration with icodextrin exchange in automated peritoneal dialysis patients? Perit Dial Int 2006; 26:336-40.

7. Venturoli D, Jeloka TK, Ersoy FF, Rippe B, Oreopoulos DG. The variability in ultrafiltration achieved with icodextrin, possibly explained. Perit Dial Int 2009; 29:415-21.

8. Jenkins SB, Wilkie ME. An exploratory study of a novel peritoneal combination dialysate $(1.36 \%$ glucose $/ 7.5 \%$ icodextrin), demonstrating improved ultrafiltration compared to either component studied alone. Perit Dial Int 2003; 23:475-80.

9. Freida P, Galach M, Filho JC, Werynski A, Lindholm B. Combination of crystalloid (glucose) and colloid (icodextrin) osmotic agents markedly enhances peritoneal fluid and solute transport during the long PD dwell. Perit Dial Int 2007; 27:267-76.

10. Parikova A, SmitW, Struijk DG, Krediet RT. Analysis of fluid transport pathways and their determinants in peritoneal dialysis patients with ultrafiltration failure. Kidney Int 2006; 70:1988-94. Epub 2006 0ct 11.

11. Williams JD, Craig KJ, Topley N, Williams GT. Peritoneal dialysis: changes to the structure of the peritoneal membrane and potential for biocompatible solutions. Kidney Int Suppl 2003; 84:158-61.

12. Wang $\mathrm{T}$, Heimburger $\mathrm{O}$, Cheng $\mathrm{H}$, Waniewski J, Bergstrom $\mathrm{J}$, Lindholm B. Effect of increased dialysate fill volume on peritoneal fluid and solute transport. Kidney Int 1997; 52: 1068-76.

13. Durand P, Chanliau J, Gamberoni J, Hestin D, Kessler M. Intraperitoneal hydrostatic pressure and ultrafiltration volume in CAPD. Adv Perit Dial 1993; 9:46-8.

14. Rusthoven $E$, van der Vlugt ME, van Lingen-van Bueren LJ, van Schaijk TC, Willems HL, Monnens LA, et al. Evaluation of intraperitoneal pressure and the effect of different osmotic agents on intraperitoneal pressure in children. Perit Dial Int 2005; 25:352-6.

15. Rusthoven E, Krediet RT, Willems HL, Monnens LA, Schroder $\mathrm{CH}$. Peritoneal transport characteristics with glucose polymer-based dialysis fluid in children. J Am Soc Nephrol 2004; 15:2940-7.

16. Geranton F, Chantrel F, Bouiller M, Muller S, Kolb I, Moulin $\mathrm{B}$, et al. Prediction of colloid osmotic pressure in renal patients. Clin Nephrol 2000; 53:269-75.

17. Duncan A, Young DS. Measurements of serum colloid osmotic pressure are of limited usefulness. Clin Chem 1982; 
28:141-5.

18. Weisberg HF. Osmotic pressure of the serum proteins. Ann Clin Lab Sci 1978; 8:155-64.

19. Konings CJ, Kooman JP, Schonck M, Gladziwa U, Wirtz J, Van Den Wall Bake AW, et al. Effect of icodextrin on volume status, blood pressure and echocardiographic parameters: a randomized study. Kidney Int 2003; 63:1556-63.

20. Davies SJ, Garcia Lopez E, Woodrow G, Donovan K, Plum $\mathrm{J}$, Williams $\mathrm{P}$, et al. Longitudinal relationships between fluid status, inflammation, urine volume and plasma metabolites of icodextrin in patients randomized to glucose or icodextrin for the long exchange. Nephrol Dial Transplant 2008; 23:2982-8. Epub 2008 May 2.

21. Garcia-Lopez E, Werynski A, Heimburger 0, Filho JC, Lindholm B, Anderstam B. Rate of synthetic oligosaccharide degradation as a novel measure of amylase activity in peritoneal dialysis patients. Perit Dial Int 2008; 28: 296-304.

22. Lam MF, Tse KC, Yip PS, Lui SL, Chan TM, Lo WK, et al. Retroperitoneal leakage as the cause for acute ultrafiltration failure and its associated risk factors in peritoneal dialysis. Perit Dial Int 2008; 28(Suppl 4):S7. 